abnormal Valsalva ratio (in 18 patients, $p<0.001$ at both comparisons, McNemar test). Abnormal handgrip test (in 73 patients) was found significantly more often than an abnormal fall in blood pressure after standing up (in 3 patients, $p<0.001$, McNemar test). Abnormal blood pressure tests were present only if at least one of the heart rate tests was abnormal.

Data were analysed according to two different protocols. By the EURODIAB protocol 38 patients $(18 \%$ ) had false negative results: they had normal $30 / 15$ ratio and normal blood pressure response to standing; however, in 18 patients one, and in 20 patients, more than one of the three other tests were abnormal.

According to our protocol, heart rate response to deep breathing and 30/15 ratio were examined. In this case, only three patients $(1.5 \%)$ showed false-negative results, only the Valsalva ratio being abnormal. Both heart rate responses to deep breathing and standing were abnormal in 45 of our 206 patients. However, 32 of these 45 patients had abnormal results in at least one of the three other tests as well, indicating moderate to severe autonomic involvement. Thus, evaluation of these two tests also seems to provide some possibility for the assessment of severity of autonomic dysfunction.

Both protocols examined the 30/15 ratio. Evaluation of heart rate response to deep breathing instead of blood pressure response to standing seems to be a useful alternative way for screening autonomic function. Both tests equally require $60 \mathrm{~s}$.

Recently, we suggested evaluating heart rate responses to deep breathing and standing as the first step of diagnosis, as part of our rational diagnostic model for the assessment of autonomic function [3]. Indeed, for screening purposes in large epidemiological studies, examination of these two tests heart rate responses to deep breathing and standing - seems to be sufficient.

Yours sincerely,

P. Kempler, Zs. Kerényi, Gy. Tamás

\section{References}

1. EURODIAB IDDM Complications Study Group (1994) Microvascular and acute complications in IDDM patients: the EURODIAB IDDM Complications Study. Diabetologia 37: 278-285

2. Ewing DJ, Clarke BF (1982) Diagnosis and management of diabetic autonomic neuropathy. BMJ 285: 916-918

3. Kempler P, Váradi A, Tamás Gy (1990) Which battery of cardiovascular autonomic function test - suggestion for a rational diagnostic model. Diabetologia 33: 650

\title{
Prevalence of maternally inherited diabetes and deafness in diabetic populations in the Netherlands
}

\begin{abstract}
Dear Sir,
Previously we described a mutation in the mitochondrial encoded tRNA ${ }^{\text {Leu(UUR) }}$ gene at position 3243 which cosegregated with non-insulin-dependent diabetes mellitus (NIDDM) and an associated deafness [1]. Additional publications have corroborated this finding [2-4]. Furthermore, we found that this mutation, which is also found in patients with mitochondrial myopathy, encephalopathy, lactic acidosis and strokelike episodes (MELAS), is most likely the only mutation responsible for this subtype of diabetes, for which we proposed the name: Maternally Inherited Diabetes and Deafness (MIDD) [4]. Recently the prevalence of this mutation in selected French pedigrees with multiplex NIDDM was reported to be approximately $2 \%$ [5]. Katagiri et al. [2] showed that the prevalence of this mitochondrial tRNA ${ }^{\text {Leu(UUR) }}$ mutation in a Japanese group of subjects selected for maternal inheritance of diabetes is slightly more than $1 \%$. Another study by Kadowaki et al. [3] indicated a prevalence of respectively $6 \%$ and $2 \%$ in Japanese patients with familial IDDM or NIDDM.

In this letter we present the prevalence of the tRNA ${ }^{\mathrm{Leu}(\mathrm{UUR})}$ gene mutation in two NIDDM populations in the Netherlands: one population consisting of 473 randomly selected NIDDM patients from three population-based studies in the Netherlands (The 'Hoorn Study', the 'Zutphen Study' and the 'Rotterdam Study'). All patients in this group were of Caucasian origin and over 40 years of age. Diabetes was diagnosed by oral glucose tolerance test according to World Health Organi-
\end{abstract}

Corresponding author: Dr. J. A. Maassen, Department of Medical Biochemistry, Wassenaarseweg 72, P.O. Box 9503, NL2300 RA Leiden, The Netherlands zation criteria. Diabetic patients with marked insulinopenia were excluded from the present study, i.e. when requiring insulin treatment within one year after diagnosis of diabetes.

The other group comprised 28 pedigrees from the diabetes clinic of the University Hospital, Leiden, selected for the presence of NIDDM and deafness in at least two generations, and with a mode of inheritance agreeing with a maternal one. Total DNA isolation from leucocytes and detection of the 3243 mutation in the mitochondrial DNA was as described earlier [4].

In the population-based group we detected two subjects out of 473 carrying the mutation, A020 and A135 (pedigrees shown in Fig. 1). Upon further investigation of these two pedigrees it appeared that individual A020 (age 27 years) suffers from a growth hormone deficiency. Her brother, mother, ma-
A

$\mathrm{A} 020$

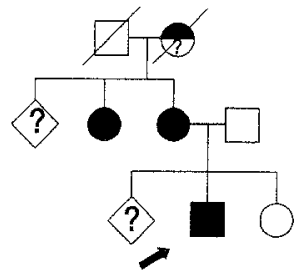

B

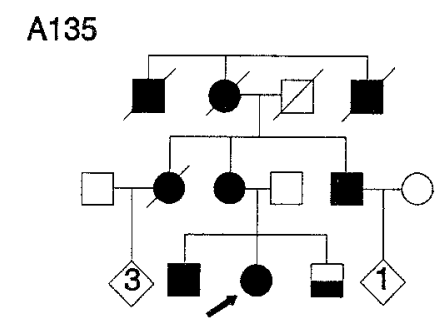

Fig. 1. Relevant parts of pedigrees. A. Patient A020. B. patient A135. Probands are indicated with an arrow. $\odot$ NIDDM: $\odot$ deafness; NIDDM and deafness; ? unknown 
ternal aunt and grandmother do have NIDDM and/or deafness (Fig. 1). It is unclear whether the growth hormone deficiency is related to the mutation in the mtDNA, but it is interesting that another report has also presented evidence for growth hormone deficiency in an individual with the 3243 mutation [6]. Other reports frequently indicate a short stature in patients with the 3243 mutation. These findings suggest that the tRNA $^{\text {Leu(UUR) }}$ mutation may exhibit three distinct but somewhat overlapping clinical phenotypes: MELAS, diabetes mellitus and deafness (MIDD) and growth hormone deficiency.

In the second group the mutation was found in three probands from unrelated pedigrees. This result shows that when stringent selection criteria are applied, the prevalence of this mutation can be as high as $10 \%$ and further corroborates the association of the MIDD subtype of diabetes and the 3243 mtDNA mutation.

We conclude that in the Netherlands the prevalence of this mutation in the average NIDDM population, lacking diabetic patients with marked insulinopenia, is low and less than $0.5 \%$. No evidence of regional clustering is seen. In addition, it should be recognized that the study of subjects from selected families with diabetes $[2,3,5$ and the present data] gives a marked overestimation of the prevalence of this gene defect in NIDDM patients.

Sincerely yours,

L. M.'t Hart, H. H. P. J. Lemkes, R. J. Heine, R. P. Stolk,

E. J. M. Feskens, J. J. Jansen, F.E.E. van der Does,
D. E. Grobbee, D. Kromhout, J. M. W. van den Ouweland, J. A. Maassen

\section{References}

1. van den Ouweland JMW, Lemkes HHPJ, Ruitenbeek W et al. (1992) Mutation in mitochondrial tRNA ${ }^{\text {Leu(UUR) }}$ gene in a large pedigree with maternally transmitted type II diabetes mellitus and deafness. Nature Genetics 1: 368-371

2. Katagiri H, Asano T, Ishihara H et al. (1994) Mitochondrial diabetes mellitus: prevalence and clinical characterization of diabetes due to mitochondrial tRNA ${ }^{\text {Leu(UUR) }}$ gene mutation in Japanese patients. Diabetologia 37: 504-510

3. Kadowaki T, Kadowaki H, Mori Y et al. (1994) A subtype of diabetes mellitus associated with a mutation in the mitochondrial gene. N Eng J Med 330: 962-968

4. van den Ouweland JMW, Lemkes HHPJ, Trembath RC et al. (1994) Maternally inherited diabetes and deafness (MIDD) is a distinct subtype of diabetes and associates with a single point mutation in the mitochondrial tRNA ${ }^{\text {Leu(UUR) }}$ gene. Diabetes 43: 746-751

5. Vionnet N, Passa P, Froguel P (1993) Prevalence of mitochondrial gene mutations in families with diabetes mellitus. Lancet $342: 1429-1430$

6. Matsuzaki M, Izumi T, Ebato K et al. (1991) Hypothalamic GH deficiency and galastic seizures in a 10-year-old girl with MELAS. No To Hattatsu 23: 411-416

\section{Book review}

B Frier, M Fisher: Hypoglycaemia and diabetes - Clinical and Physiological Aspects. Edward Arnold London; 1993, 416 pp, 140 figures, 47 tables, price $£ 95.00$ ISBN 0340554215

All physicians concerned with diabetes care know that "patients who have to take insulin nearly all agree that their greatest problem is hypoglycaemia" (Malins 1968). The problems range from a minor nuisance to a major disruption of lifestyle and even death. Intensive diabetes control has increased the problem of severe hypoglycaemia by approximately threefold.

An authoritative new book on this subject is therefore very appropriate and this is a superb book which any serious diabetes specialist should study carefully. It skilfully combines pathological and physiological consequences of hypoglycaemia with extensive clinical accounts of every aspect of the problem. The clinician will be fascinated by developments in biochemistry and genetics, which may explain varying effects on different parts of the brain while, on the other hand, practical problems of driving or those of the diabetic patient in prison are encompassed. Readers will find here everything they need to know whether in relation to the law or the latest information on hypoglycaemic unawareness, and there is a unique chapter on the historical aspects as well.

If I have any criticism, it is the absence of a clearly written straightforward clinical description of the causes, effects, treatment and prevention of hypoglycaemia: the information is all in this book but perhaps of necessity in a text of such substantial depth and with such an extensive bibliography, that the descriptions are scattered throughout the different chapters. The book has the stamp of authority throughout: most of the authors are well known, while several have devoted most of their professional lives to the study of hypoglycaemia and have deservedly international reputations. It is a tribute to the editors that they have all contributed such substantial chapters.

This book will clearly continue to be the most authoritative account of hypoglycaemia in diabetes for many years to come and deserves to be successful.

PJ Watkins

London 